Palacios Picos, A.; López-Pastor, V.; Fraile Aranda, A. (2019) Perception Questionnaire of Teaching Competences in Physical Education. Revista Internacional de Medicina y Ciencias de la Actividad Física y el Deporte vol. 19 (75) pp. 445-461 Http://cdeporte.rediris.es/revista/revista75/artdeterminante1038.htm DOI: $10.15366 /$ rimcafd2019.75.005

\title{
ORIGINAL
}

\section{CUESTIONARIO DE PERCEPCIÓN DE COMPETENCIAS DOCENTES DE EDUCACIÓN FÍSICA}

\section{PERCEPTION QUESTIONNAIRE OF TEACHING COMPETENCES IN PHYSICAL EDUCATION}

\author{
Palacios Picos, A. ${ }^{1}$; López-Pastor, V. ${ }^{1}$ y Fraile Aranda, A. ${ }^{2}$ \\ ${ }^{1}$ Catedrático de Escuela Universitaria. Universidad de Valladolid. Facultad de Educación de \\ Segovia (España), palacios@psi.uva.es \\ ${ }^{2}$ Catedrático de Universidad. Universidad de Valladolid. Facultad de Educación de Segovia \\ (España),vlopez@mpc.uva.es \\ ${ }^{3}$ Catedrático de Universidad. Universidad de Valladolid. Facultad de Educación y Trabajo Social \\ de Valladolid (España), antonio.fraile@uva.es
}

FINANCIACIÓN: Este estudio se ha llevado a cabo dentro del proyecto de I+D+i: "La competencias docentes en la formación inicial del profesorado de educación física". Convocatoria de noviembre de 2013 del Programa Estatal de Investigación, Desarrollo e Innovación Orientada a los Retos de la Sociedad, en el marco del Plan Estatal de Investigación Científica y Técnica y de Innovación 2014-2017. Referencia: EDU 2013-42024-R. Duración: 3 años (2014-2017).

Código UNESCO / UNESCO code: 5801.07 Métodos Pedagógicos / Teaching methods.

Clasificación del Consejo de Europa / Council of Europe classification: 5. Didáctica y metodología / Didactic and methodology.

Recibido 30 de octubre de 2017 Received October 30, 2017

Aceptado 25 de agosto de 2018 Accepted August 25, 2018

\section{RESUMEN}

La incorporación de las competencias en la universidad española es un elemento clave de su calidad. Sin embargo, no se dispone de instrumentos adecuados ni suficientes para su medida en el campo concreto de la formación inicial del profesorado de Educación Física. El principal objetivo de este trabajo es diseñar y validar una escala para medir la percepción de competencias docentes de este alumnado. Para su construcción y validación, se ha seguido una metodología fundamentada en la Teoría Clásica de Test incidiendo especialmente en el cálculo de la fiabilidad y de la validez de la escala. La muestra para su validación es de 1713 estudiantes y egresados (1240 estudiantes, 473 egresados), pertenecientes a 20 universidades españolas. La escala final presenta una 
estructura factorial de cuatro factores principales y 22 ítems con los que se alcanza una alta fiabilidad y una adecuada validez de contenido y de constructo.

PALABRAS CLAVE: Competencias Docentes, Formación Inicial del Profesorado, Educación Física, Validación de Escalas, Análisis Factorial Confirmatorio.

\begin{abstract}
The incorporation of competences in Spain is a key element of its quality. However, there are no instruments available to measure them in the specific field of Physical Education Teacher Education. The main objective of this study is to design and validate a scale to measure the perception of the teaching competences of these students. For its construction and validation, a methodology based on the Theory of the Classic Test was followed, with special emphasis on the calculation of the reliability and validity of the scale. The sample for its validation is 1713 students and graduates (1240 students, 473 graduates), belonging to 20 Spanish universities. The final scale presents a factorial structure of four main factors and 22 items with which high reliability and adequate content and construct validity are achieved.
\end{abstract}

KEY WORDS: Teaching competencies, Pre-service teacher education, Physical Education, scales validation, Factorial Confirmatory Analysis.

\title{
INTRODUCCIÓN
}

La nueva sociedad del conocimiento requiere innovaciones y cambios en las formas tradicionales de formación, producción, comunicación e información (Delors, 1996). El bienestar de los ciudadanos, el dinamismo de la economía y la profundización en la participación democrática dependen, en gran medida, de la forma en que las sociedades incorporen estos cambios y asuman las transformaciones sociales que conllevan, siendo un aspecto esencial para ello partir del bienestar del propio docente (Marchesi, 2005).

El Espacio Europeo de Enseñanza Superior (EEES) tiene como finalidad desarrollar políticas que favorezcan una educación en constante cambio, así como una transformación trascendente del diseño de los nuevos estudios de Grado dirigidos a la formación del profesorado. La reforma universitaria que surge a partir del proceso de Bolonia exige una nueva ordenación de la actividad académica para abordar, en el marco social de la información y del conocimiento, los retos derivados de la innovación (Garmendia, 2009). Como consecuencia de este proceso de convergencia hacia el EEES, la incorporación de las competencias en los estudios universitarios resulta un elemento básico para la formación en una sociedad cambiante, que reformula sus demandas constantemente y que, a su vez, aspira a profesionalizar la formación universitaria acercando la Universidad a la sociedad y al mundo laboral (Palmer, Montaño y Palou, 2009). Por otro lado, como se apunta en el Informe Delors (1996), en lugar de cualificación, la sociedad reclama cada vez más la 
competencia, que combina la capacitación y cualificación adquirida por la formación técnica y profesional, con el comportamiento social, la aptitud para el trabajo en equipo, la iniciativa, el gusto por el riesgo... En el concepto de competencia se integra el saber, el saber hacer y el saber ser (Delors, 1996).

El concepto de competencia tiene varios referentes (Le Boterf, 2000; Martín y Moreno, 2007; Perrenoud, 2004; Zabalza, 2003), que consideran que dicho concepto supone la selección y combinación pertinente de conocimientos, habilidades, destrezas, actitudes, valores y normas que permiten dar respuesta a una situación compleja en un contexto determinado. Para Tejada (2005) "una competencia representa un conjunto de conocimientos, procedimientos y actitudes combinados, coordinados e integrados, en el sentido que el individuo ha de saber hacer y saber estar para el ejercicio profesional", (p.7). Hace referencia a los cuatro saberes de la definición de competencias: conocimiento teórico de un ámbito académico del ámbito profesional ("saber"), aplicar los conocimientos a situaciones profesionales concretas ("saber hacer"), características y actitudes personales hacia sí mismo, hacia los demás y hacia la profesión ("saber ser") y el conjunto de actitudes y habilidades interpersonales que permiten interactuar en el entorno profesional ("saber estar")". Ser profesionalmente competente equivale a ser capaz de desempeñar de forma suficiente y adecuada diferentes tareas profesionales relacionadas con dichas competencias. Del mismo modo, la competencia supone la capacidad de responder con éxito a las exigencias personales y sociales que nos plantea una actividad o una tarea cualquiera en el contexto del ejercicio profesional (Rué, 2007).

Los objetivos de los nuevos planes de estudio deberán proporcionar una formación universitaria en la que se integren de forma armónica las competencias genéricas básicas, las competencias transversales relacionadas con la formación integral de las personas y las competencias más específicas, que posibiliten una orientación profesional que permita a los titulados una integración en el mercado de trabajo (MECD, 2003). Existen diferentes modelos que agrupan las competencias consideradas fundamentales desde el punto de vista del desempeño profesional. Para el presente estudio se han considerado las propuestas realizadas por Pereda y Berrocal (1999) y Solanes, Núñez y Rodríguez (2008), que diferencian entre dos tipos de competencias: (a) Competencias genéricas o transversales: aquéllas que pueden ser comunes y se refieren a características generales de las personas; (b) Competencias específicas: aquéllas relacionadas con un área de trabajo concreto (como el caso del profesorado de Educación Física).

En el ámbito específico de la formación del profesorado de Educación Física, el futuro docente deberá adquirir una serie de competencias que le permitan atender y resolver situaciones problemáticas relacionadas con una práctica generadora del cambio, a través de una mejora profesional, con la reconstrucción posible de la realidad y de un posicionamiento personal y profesional efectivo. Para ello, es preciso superar un tipo de perspectiva academicista y eficientista-mercantilista del trabajo docente, impulsando un modelo de enseñanza-aprendizaje basado en la reflexión y en la autocrítica sobre la praxis, que lleve a ese docente a un cuestionamiento de su propio ejercicio profesional (Fraile, 2004). En las "competencias docentes específicas" el profesor debe conocer la materia que 
enseña y cómo debe enseñarla, para lo cual deberá tener unos conocimientos epistemológicos propios de la Educación Física. En consecuencia, requiere conocimientos técnicos-científicos y educativos de su actividad profesional y capacidades de análisis y reflexión, de comprensión y aplicación del proceso (Fraile y Aparicio, 2015a, 2015b).

La investigación en torno a las competencias profesionales se inician en los años 70 , dentro de los enfoques tecnológicos y conductistas de la formación del profesorado, según tendencias de modelos tales como el CBTE (Competency Based Teacher Education) (Navio, 2005). Del mismo modo, en los países desarrollados se han diseñado documentos que describen y estructuran las capacidades y habilidades para los diversos grupos o familias profesionales que el trabajador ha de adquirir y los criterios para valorar el nivel de perfeccionamiento alcanzado. Algunos ejemplos de ello son: "USA National Skills Standards" en Estados Unidos; "General National Vocational Qualifications" en el Reino Unido; "National Competency Standards at Technical and Further Education" en Australia (Corominas, 2001). Del mismo modo, en el contexto internacional, uno de los trabajos a destacar sobre competencias docentes es el realizado por las profesoras Bourgonje y Tromp (OXFAM NOVID, 2011), investigadoras en el campo de la educación y del desarrollo de la Universidad de Ámsterdam (Países Bajos), sobre los educadores de calidad, en el que analizan las competencias y estándares para docentes de diferentes países.

El campo de la evaluación de competencias docentes es novedoso en nuestro país, tanto en lo relativo a las docentes generales como de las competencias docentes específicas de Educación Física. En este último campo, los estudios de evaluación de competencias se han centrado fundamentalmente en la revisión de qué tipo de competencias están presentes en la formación del profesorado de Educación Física (Baena y Granero, 2012; Boned, Rodríguez-Romo, Mayorga y Merino, 2006; Díaz del Cueto, 2009; Gallardo, 2006; Gallego-Ortega y RodríguezFuentes, 2017; Hernández, 2007; Lleixá, Robert y Batalla, 2010; Lleixá, Torralba, Abrhao, 2010; Romero, 2009). Como aportación concreta sobre evaluación de competencias cabe citar el trabajo de Baena, Granero y Martínez (2015) sobre la validación española de la Escala de Evaluación de Competencias Docentes (Evaluation of Teaching competencies Scale-ETCS), llevada a cabo con el objeto de medir las competencias del profesorado de Educación Física de Secundaria. Así como el trabajo de Martínez-Mínguez (2016), cuyos objetivos se concretan en analizar la influencia del desarrollo de un proyecto de aprendizaje tutorado, con equipos de profesorado mixtos universidad-maestros de escuela y en la elaboración de buenas prácticas sobre la percepción de competencias docentes. El instrumento utilizado para la evaluación es denominado: "Escala de Autopercepción de Competencias Profesionales Psicomotrices" (EAACPP), que analiza cinco competencias: organización, evaluación, trabajo en equipo, programación, ambiente y difusión.

En los niveles educativos universitarios, Salcines-Talledo, González-Fernández, Ramírez-García y Martínez-Mínguez (2018) han desarrollado y validado una escala de auto-percepción de competencias transversales y profesionales para estudiantes, en la que se incluye una escala específica para la formación inicial 
del profesorado de Educación Física (FIPEF). Por otra parte, Moreno-Murcia, Silveira y Belando (2015) han elaborado un cuestionario para evaluar el desempeño y las competencias del profesorado universitario (Cuestionario de Evaluación del Desempeño de la Enseñanza-CEID), que aplican a una muestra de estudiantes universitarios. Sus resultados muestran una estructura factorial de tres dimensiones (planificación, desarrollo y resultado) con una adecuada fiabilidad y validez. Por su parte, Castejón-Oliva, Santos-Pastor y Palacios-Picos (2015) validan un cuestionario para evaluar las percepciones de los estudiantes en relación con la metodología participativa y la evaluación formativa recibida.

Por último, en Aparicio y Fraile (2015) se analiza la adquisición de competencias docentes interpersonales a partir del desarrollo de un programa de expresión corporal con futuros docentes de Educación Física. Para la evaluación de la percepción de competencias del alumnado los autores utilizan la "Escala de valoración de competencias interpersonales del docente de Educación Física" (EcoiDEF). Su finalidad era valorar las competencias genéricas de estudiantes universitarios, para lo cual utilizan 33 ítems que corresponden con las siguientes competencias interpersonales: trabajo en equipo, comunicación con expertos de otras áreas, habilidades en las relaciones interpersonales, apreciación de la diversidad y la multiculturalidad, capacidad de autocrítica, crítica y compromiso ético. Para la elaboración de dicho instrumento se tomaron como referentes a Solanes et al, (2008) y Corominas et al, (2006).

\section{OBJETIVOS}

La intención de este estudio es desarrollar un instrumento de medida sobre la percepción de competencias docentes específicas de Educación Física, para ser aplicado en estudiantes en formación. Concretamente, nuestros objetivos son:

1-Diseñar una escala para medir la percepción de competencias del alumnado de las titulaciones de FIPEF.

2-Comprobar sus propiedades psicométricas, con especial incidencia en la constatación de su validez de contenido y de su validez de constructo.

\section{MÉTODO}

\section{MUESTRA}

La muestra utilizada para validar el presente cuestionario está formada por 1.713 estudiantes y egresados (1.240 estudiantes, 473 egresados), pertenecientes a 20 centros universitarios de 20 ciudades españolas (Tabla 1).

Tabla 1. Origen de los centros universitarios

\begin{tabular}{lcc} 
& Frecuencias & Porcentaje \\
\hline Sevilla & 220 & 12,8 \\
Murcia & 173 & 10,3 \\
Albacete & 164 & 9,6 \\
Barcelona & 158 & 9,2 \\
Lleida & 124 & 7,2
\end{tabular}




\begin{tabular}{lcc} 
Segovia & 119 & 6,9 \\
Granada & 99 & 5,8 \\
León & 93 & 5,4 \\
Huesca & 93 & 5,4 \\
Vitoria-Gasteiz & 89 & 5,2 \\
Valencia & 82 & 4,8 \\
Valladolid & 69 & 4,0 \\
Santander & 64 & 3,7 \\
Madrid & 52 & 3,0 \\
Córdoba & 44 & 2,6 \\
La Laguna & 42 & 2,5 \\
Zamora & 23 & 1,3 \\
Salamanca & 3 & 0,2 \\
Zaragoza & 2 & 0,1 \\
\hline Total & 1713 & 100
\end{tabular}

La selección de los participantes se realizó mediante un muestreo no probabilístico, tomando como referencia las universidades pertenecientes a una Red Nacional de Evaluación Formativa en Educación Superior. La toma de datos fue realizada por docentes debidamente entrenados y con el permiso expreso de todos los participantes.

Del total de 1.240 estudiantes, 793 (64\%) cursaban el Grado de Educación Primaria (itinerario de Educación Física) y los restantes 447 (36\%) el Grado en Ciencias de la Actividad Física y del Deporte (CAFYD). En todos los casos, en el momento de la toma de datos cursaban el último curso del grado, al final del segundo semestre. En cuanto a los egresados, 137 de los 473 (29\%) trabajaban como docentes en colegios públicos o concertados, siendo su experiencia docente de un año por término medio; el restante $71 \%$ o no trabajaba o lo hacían en otros trabajos no relacionados con la docencia; el $83 \%$ habían terminado sus estudios superiores en los últimos tres últimos cursos escolares. El $76 \%$ de los encuestados tienen menos de 30 años, un 14\% entre 31 y 35 años y sólo un $9 \%$ más de 36 años. De ellos, el 57\% son hombres y el $43 \%$ mujeres.

Además, en la validación del contenido de la prueba han participado 8 juecesevaluadores de diferentes universidades españolas. Su edad media es de 43 años y su experiencia profesional por término medio es de 15 años de diferentes categorías profesionales. Todos ellos son profesores universitarios con docencia relacionada con las competencias que se pretenden medir.

\section{PROCEDIMIENTO}

\section{Primera fase: Validación de expertos de los contenidos del cuestionario}

Siguiendo las indicaciones de Carretero-Dios y Pérez (2005), un grupo de investigadores de la Red Nacional de Evaluación en Educación Física fue elaborando los primeros borradores del cuestionario en una primera etapa. En párrafos anteriores se ha señalado las diferencias entre dos grandes tipos de competencias: (a) genéricas o transversales, aquéllas que son comunes y se refieren a características generales de las personas; (b) específicas, aquéllas relacionadas con un área de trabajo concreto; en este caso están referidas al ámbito del profesorado de Educación Física. Por ello, en un segundo paso, las competencias 
específicas a tener en cuenta en la formación inicial del profesorado de Educación Física se han desglosado en dos grandes tipos: (1) las competencias docentes generales; y, (2) las competencias docentes propias de la Educación Física. En una primera versión del cuestionario se utilizó el listado de competencias comunes a todos los Grados de Educación Primaria, así como las competencias específicas del maestro especialista en Educación Física del Libro Blanco (ANECA, 2005b), así como el listado de competencias recogidas en el Libro Blanco para el título de Grado de CAFYD (ANECA, 2005a).

Con la primera versión del cuestionario se llevó a cabo una revisión de 8 jueces expertos, especializados en la FIPEF, tanto de mención de Educación Física en Primaria como del Grado de CAFYD, con un mínimo de 15 años de experiencia en FIPEF y reconocido prestigio docente e investigador. Tras el proceso de revisión y análisis de las valoraciones aportadas por los jueces expertos se obtuvo una segunda versión del cuestionario, tras agrupar, eliminar, depurar y modificar ítems, quedando con un total de 27 ítems, de los 38 iniciales.

\section{Segunda fase: depuración de la escala inicial}

La segunda versión del cuestionario se aplicó a una muestra aleatoria de 85 alumnos de último curso de las dos titulaciones de grado específicas, de cuatro universidades diferentes. En cada una de las titulaciones los investigadores responsables repartieron un cuestionario a cada alumno, solicitándoles que indicaran los ítems cuyo significado no entendían bien o podían generar problemas o errores de comprensión. También se les solicitó que marcaran las expresiones que eran confusas y que podrían entenderse mejor si se plantearan de otra forma. Tras realizar ese proceso individual de revisión, se realizó una entrevista grupal con ellos, hablando de los problemas de comprensión encontrados y triangulando las valoraciones de cada alumno con las del resto. A partir de todas las revisiones y valoraciones aportadas, se revisó el cuestionario y se obtuvo una nueva versión del mismo, compuesta por 22 ítems, que fue considerada la escala final.

\section{Tercera fase: Análisis psicométrico de la escala final}

Para el cálculo de los valores psicométricos de esta escala final de 22 ítems se ha utilizado el programa estadístico SPSS v.23 y el programa Lisrel 8.8. Con el primero se han realizado los correspondientes análisis exploratorios de los ítems buscando los posibles valores fuera de rango. Estos valores, junto con los valores perdidos (tanto los definidos por el sistema como los definidos por el usuario) fueron escasos y nunca superaron el $1 \%$ de los totales. Además, se obtuvo una primera matriz de factores. Tras este primer Análisis Factorial Exploratorio (AFE), sus factores fueron sometidos a un Análisis Factorial Confirmatorio (AFC) con el programa Lisrel 8.8. Para este cálculo se obtuvieron las correlaciones policóricas, dada la naturaleza ordinal de las variables intervinientes según el criterio de Olsson (1979). El método de estimación de los parámetros del modelo fue el de distribución asintóticamente libre, que la hace eficiente para cualquier distribución de las variables, no haciendo necesario el supuesto de normalidad multivariante (Batista y Coenders, 2000). Tras dichos 
cálculos se obtuvieron los valores de fiabilidad final de las diferentes escalas, producto de este AFC y de la escala total.

\section{RESULTADOS}

\section{VALIDEZ DE CONSTRUCTO Y FIABILIDAD FINAL}

En un primer AFE se obtuvieron cuatro factores principales que explicaban un $53 \%$ de la varianza total (Tabla 2). Los indicadores de la pertinencia y validez de este análisis presentaron valores adecuados $(\mathrm{KMO}=, 95$; Chi-cuadrado=12492,2; $\mathrm{p}=, 00)$. 


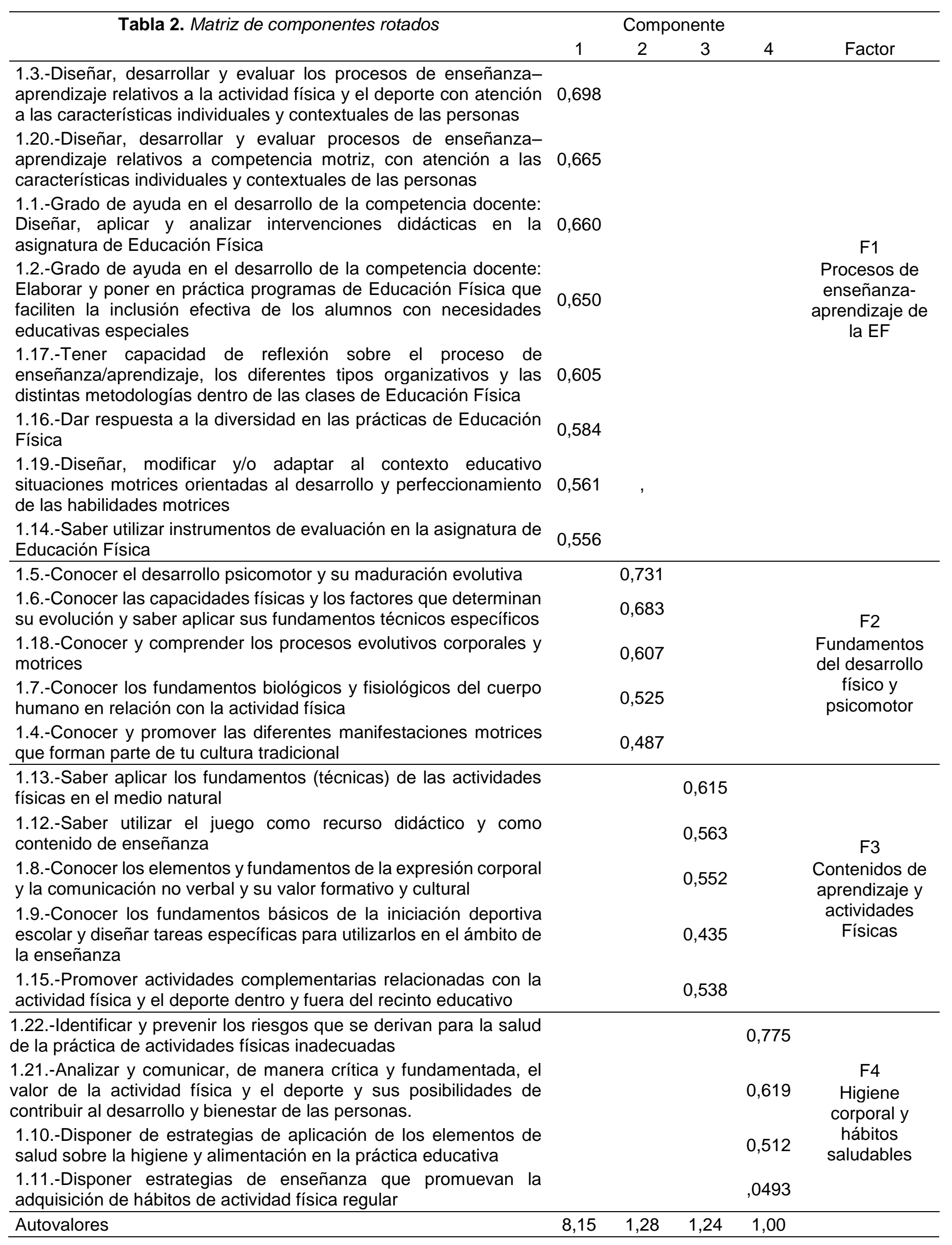




\begin{tabular}{|c|c|c|c|}
\hline \% Varianza explicada & $37,08 \quad 5,82$ & 5,66 & 4,56 \\
\hline \% Varianza explicada acumulado & $37,08 \quad 42,91$ & 48,58 & 53,14 \\
\hline $\mathrm{KMO}$ & \multicolumn{3}{|c|}{0.95} \\
\hline Prueba Esfericidad de Barlett & \multicolumn{3}{|c|}{ Chi-cuadrado $=12492,67 ; \mathrm{gl}=231 ; \mathrm{sig}=0,00$} \\
\hline
\end{tabular}

El primer factor (F1) explica el $37 \%$ de la varianza total de la prueba y tiene pesos elevados en aspectos relacionados con el diseño, desarrollo y evaluación de los procesos de enseñanza-aprendizaje, tomando en consideración las características de los estudiantes y sus necesidades educativas especiales. Por ello, se ha denominado a este factor "Competencias relacionadas con los procesos de enseñanza-aprendizaje de la Educación Física". El segundo de los factores (F2), relacionado con los fundamentos del desarrollo físico y psicomotor, explica el $6 \%$ de la varianza total y tiene importantes correlaciones con el conocimiento del desarrollo físico y psicomotor desde una perspectiva evolutiva y en relación con la actividad física. El tercero de los factores (F3) se le ha denominado "Contenidos de aprendizaje y actividades Físicas" por el peso de ítems relacionados con dichos contenidos como son: la iniciación deportiva en la escuela, las actividades físicas en el medio natural, la expresión corporal, los juegos y las actividades físicas organizadas, bien en el ámbito educativo en general o en un contexto extraescolar. El cuarto y último de los factores (F4) se relaciona con aspectos tales como la identificación y prevención de los riesgos para la salud que se derivan de la práctica de la actividad física, las relaciones entre actividad física, alimentación, salud y bienestar personal, así como la aplicación de estrategias de adquisición de hábitos de actividad física regular. Por todo ello se le ha denominado: "Competencias relacionadas con la higiene corporal y los hábitos saludables”.

Además se consideró la existencia de un factor general ("Competencias Docentes Específicas del Profesor de Educación Física") que englobaría a estos cuatro factores. Hipótesis que se han contrastado mediante un AFC cuyos valores de ajuste se resumen en la Tabla 3.

Tabla 3. Evaluación de la estructura factorial del cuestionario (AFC)

\begin{tabular}{|c|c|c|c|c|c|c|c|}
\hline Modelo & $\begin{array}{l}\text { S-B } \text { (Chi-cuadrado) }_{\text {(gl) }}(p) \\
\text { (g) }\end{array}$ & RMSEA & NFI & NNFI & CFI & AGFI & AIC \\
\hline $\begin{array}{c}\text { Cuatro } \\
\text { subfactores y } \\
\text { un factor } \\
\text { principal }\end{array}$ & $\begin{array}{c}1458.54(200)(p \\
=0,00)\end{array}$ & 0,068 & 0,97 & 0,97 & 0,98 & 0,89 & 1617.93 \\
\hline
\end{tabular}

El modelo presenta un ajuste adecuado con valores del índice de ajuste normalizado (NFI) y no normalizado (NNFI), por encima de los mínimos establecidos para este tipo de cálculos (Bentler y Bonnett; 1980). Asimismo, el índice de ajuste comparado (CFI) de Bentler (1990) y el índice AIC (Akaike, 1987) presentaron valores adecuados. Por último, el error cuadrático medio de aproximación (RMSA) también presenta valores igualmente adecuados para este tipo de modelos (Browne y Cudeck, 1993). En la Figura 1 se presentan los valores particulares del ajuste de todos ítems y de cada uno de los factores de dicho modelo (Figura 1). 


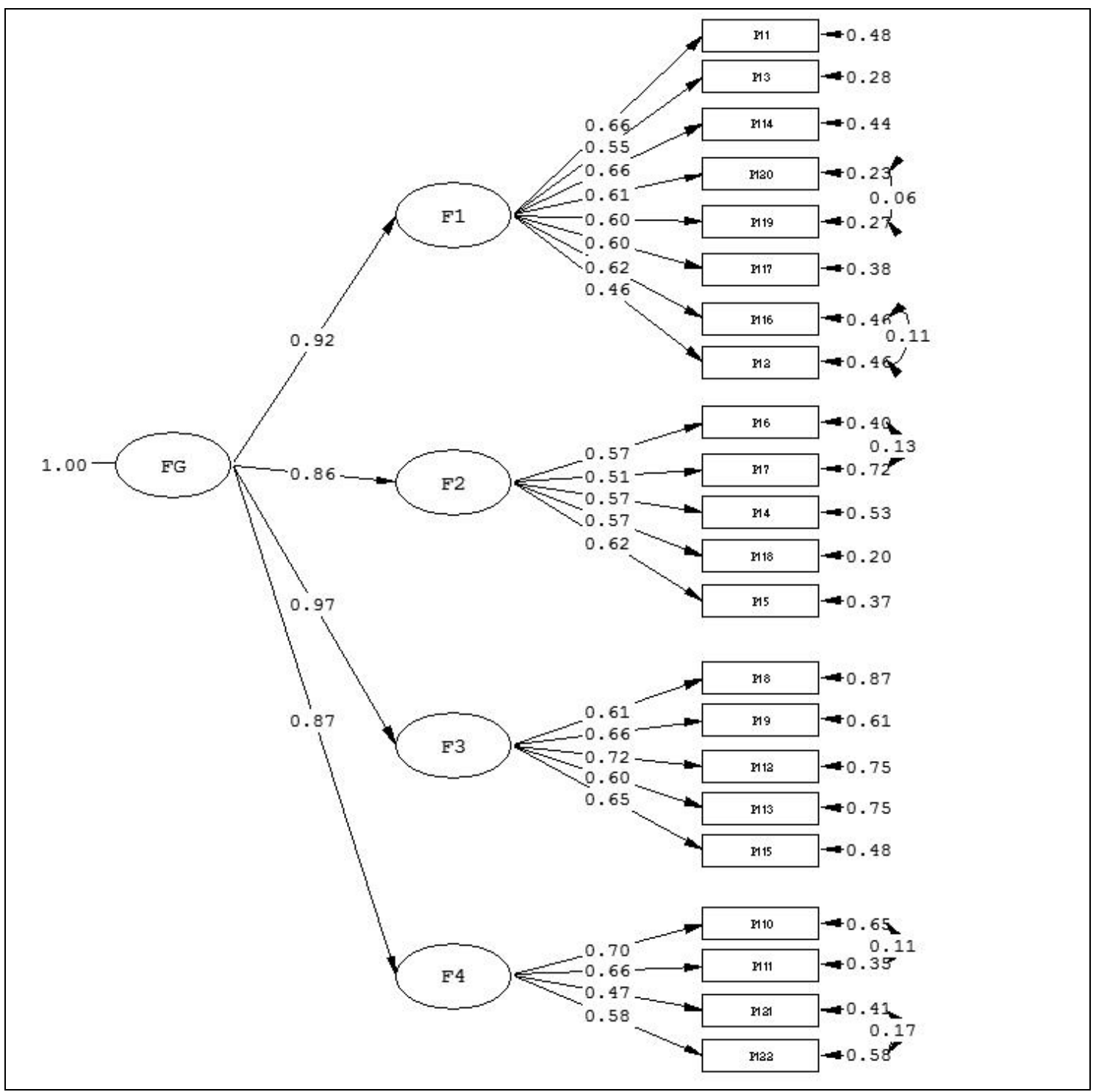

Figura 1.- Resultados del AFC

Como se puede comprobar, los 7 ítems que forman el F1 presenta coeficientes significativos; además, la fiabilidad del factor (de la subescala) es elevada (Tabla 4). Resultados que volvemos a encontrar con el resto de factores: todos los coeficientes son significativos y con valores de fiabilidad adecuados. Mención especial merece los valores de la prueba en su totalidad (factor FG), con un alfa de Cronbach elevado y con los cuatro factores con cargas factoriales igualmente altas y significativas.

Tabla 4. Estructura factorial del cuestionario y análisis de su fiabilidad

\begin{tabular}{|c|c|c|c|c|c|c|}
\hline Ítems & Factor & $\begin{array}{c}\text { Coeficientes } \\
\text { de } \\
\text { regresión }\end{array}$ & $t$ & Sig. & $\begin{array}{l}\text { Coeficiente de } \\
\text { determinación }\end{array}$ & $\begin{array}{c}\text { Alfa de } \\
\text { Cronbach }\end{array}$ \\
\hline $\mathrm{P} 11$ & \multirow{7}{*}{$\mathrm{F} 1$} & 0,66 & 240,16 & 0,02 & 0,48 & \multirow{7}{*}{0,859} \\
\hline P12 & & 0,46 & 190,61 & 0,02 & 0,31 & \\
\hline P13 & & 0,55 & 240,96 & 0,02 & 0,52 & \\
\hline $\mathrm{P} 114$ & & 0,66 & 240,51 & 0,02 & 0,50 & \\
\hline P116 & & 0,62 & 230,41 & 0,02 & 0,45 & \\
\hline P117 & & 0,60 & 240,19 & 0,02 & 0,49 & \\
\hline P119 & & 0,60 & 250,85 & 0,02 & 0,57 & \\
\hline
\end{tabular}




\begin{tabular}{|c|c|c|c|c|c|c|}
\hline P120 & & 0,61 & 260,83 & 0,02 & 0,62 & \\
\hline P14 & \multirow{5}{*}{$\mathrm{F} 2$} & 0,57 & 230,92 & 0,02 & 0,38 & \multirow{5}{*}{0,760} \\
\hline P15 & & 0,62 & 210,03 & 0,02 & 0,51 & \\
\hline P16 & & 0,57 & 20,20 & 0,02 & 0,45 & \\
\hline P17 & & 0,51 & 160,31 & 0,03 & 0,27 & \\
\hline P118 & & 0,57 & 220,42 & 0,02 & 0,62 & \\
\hline P18 & \multirow{5}{*}{ F3 } & 0,61 & 240,95 & 0,03 & 0,30 & \multirow{5}{*}{0,702} \\
\hline P19 & & 0,66 & 180,09 & 0,03 & 0,42 & \\
\hline P112 & & 0,72 & 170,93 & 0,04 & 0,41 & \\
\hline P113 & & 0,60 & 160,70 & 0,03 & 0,33 & \\
\hline P115 & & 0,65 & 180,76 & 0,03 & 0,47 & \\
\hline $\mathrm{P} 110$ & \multirow{4}{*}{ F4 } & 0,70 & 20,16 & 0,03 & 0,43 & \multirow{4}{*}{0,741} \\
\hline P111 & & 0,66 & 250,18 & 0,02 & 0,56 & \\
\hline P121 & & 0,47 & 170,64 & 0,02 & 0,35 & \\
\hline P122 & & 0,58 & 180,00 & 0,03 & 0,37 & \\
\hline Factores & Factor & $\begin{array}{l}\text { Coeficientes } \\
\text { de regresión }\end{array}$ & $\mathrm{t}$ & Sig. & $\begin{array}{l}\text { Coeficiente de } \\
\text { determinación }\end{array}$ & $\begin{array}{c}\text { Alfa de } \\
\text { Cronbach }\end{array}$ \\
\hline F1 & \multirow{4}{*}{$F G$} & 0,92 & 260,51 & 0,03 & 0,85 & \multirow{4}{*}{0,917} \\
\hline $\mathrm{F} 2$ & & 0,86 & 210,72 & 0,04 & 0,74 & \\
\hline F3 & & 0,97 & 20,58 & 0,04 & 0,93 & \\
\hline F4 & & 0,87 & 220,49 & 0,03 & 0,76 & \\
\hline
\end{tabular}

La fiabilidad de las cuatro subescalas fue aceptable en todos los casos y los índices de discriminación y correlación ítems-escala fueron superiores a lo exigible en este tipo de estudios. Considerando la escala total de competencias, existen igualmente correlaciones adecuadas entre todos los ítems y, en ningún caso, la eliminación de ítem alguno mejoraba la fiabilidad de la prueba.

\section{CONCLUSIONES}

La implantación del EEES ha supuesto un reto y una importante oportunidad de mejora para las universidades, así como una posición preponderante del concepto de "competencia", y su relación con los cuatro tipos de saberes (saber, saber hacer, saber ser y saber estar). Los nuevos planes de estudio de grado integran diferentes tipos de competencias. En las titulaciones orientadas a la formación inicial de profesorado destacan las competencias docentes generales y específicas. En las titulaciones de FIPEF ambos tipos de competencias han sido suficientemente concretadas y desarrolladas a nivel legal, pero no podemos decir lo mismo de su medida. Sabemos qué medir, pero desconocemos cómo medirlas. En este sentido, disponer de un instrumento que nos permita medir competencias se presenta como una importante necesidad. Disponer de un instrumento válido y fiable en la medida de la percepción de competencias docentes de los estudiantes de FIPEF cubre dicha necesidad y llena el vacío existente.

Tras un adecuado proceso de validación de contenido realizado por expertos en docencia universitaria y una fase piloto de comprensión de los ítems, la escala final quedó formada por 22 ítems. El primer Análisis Factorial Exploratorio (AFE) mostró una estructura de cuatro factores de segundo orden y un factor de primer orden. Esta estructura factorial es posteriormente ratificada por el Análisis 
Factorial Confirmatorio (AFC), que presentó indicadores adecuados del ajuste del modelo propuesto.

El primero de los factores ("Competencias relacionadas con los procesos de enseñanza-aprendizaje de la Educación Física") resalta la importancia para el futuro docente del conocimiento de los procesos de enseñanza-aprendizaje en educación física y la adquisición de las capacidades reflexivas de estos procesos, en un contexto de inclusión educativa en el que la atención a la diversidad adquiere una especial relevancia. El segundo de los componentes se relaciona con los "Fundamentos del desarrollo físico y psicomotor". Se trata de un conjunto de competencias centradas en el saber cómo componente competencial formadas por aspectos que se relacionan con el conocimiento del desarrollo psicomotor y los procesos de maduración evolutiva, tanto corporales como motrices; así como los fundamentos biológicos y fisiológicos del cuerpo humano en relación con la actividad física. El tercer factor ("Contenidos de aprendizaje y actividades Físicas") hace referencia a los contenidos de aprendizaje propios de la educación física y las actividades físicas organizadas que constituyen actividades sociales relevantes en estos momentos, por lo que deben formar parte de la cultura física de los estudiantes. El cuarto y último factor ("Higiene corporal y hábitos saludables") resalta la importancia para la formación del futuro docente de Educación Física de aspectos relacionados con la higiene corporal, la alimentación, el fomento de hábitos saludables y la importancia de estos y otros aspectos en el desarrollo y el bienestar personal.

Constatada la validez de contenido y la validez de constructo, se analizaron los valores de la fiabilidad tanto de la escala en su conjunto como de cada uno de sus factores. Con respecto al conjunto de la escala, el índice de fiabilidad obtenido nos permite asegurar que estamos en presencia de un instrumento con alta fiabilidad. Fiabilidad elevada que encontramos igualmente en la subescala de competencias relacionadas con los procesos de enseñanza-aprendizaje de la Educación Física. El resto de subescalas presentan valores de fiabilidad de menor cuantía pero, en todos los casos, dentro de los límites admitidos para este tipo de escalas. La totalidad de los ítems de la escala y de las subescalas presentaron valores de significación estadística en el modelo estructural; además, la eliminación de ninguno de ellos producía un aumento significativo de la fiabilidad, lo que asegura la pertinencia de su presencia en la escala final. Igualmente, las correlaciones de todos los ítems con sus correspondientes escalas presentaban valores estadísticamente significativos.

Estos datos aseguran la consistencia psicométrica de la escala final y nos permiten disponer de un instrumento sólido y robusto para la evaluación de las competencias que debe adquirir el alumnado durante su formación como docente de Educación Física. Por tanto, el instrumento podría tener varias utilidades: (a) realizar estudios sobre el grado de percepción de competencias que tiene el alumnado al finalizar su FIP; (b) ayudar a elaborar instrumentos de evaluación más detallados para poder evaluar y fomentar el desarrollo de estas competencias profesionales en las diferentes asignaturas que estén implicadas en su desarrollo; (c) ayudar a regular las competencias que deben ser consideradas en el desarrollo y defensa del Trabajo de Fin de Grado, dado que se supone que es una evidencia que debe presentar el alumno al final de su 
FIPEF, en la que demostrar que ha adquirido las competencias profesionales necesarias; (d) establecer coordinaciones entre las asignaturas encargadas de la formación general y la específica a lo largo de la FIPEF; y (e) que dichos centros universitarios puedan revisar sus planes de estudio y con ello poder llegar a incrementar los niveles de éxito académico de su alumnado.

Del mismo modo, podría ser muy útil para la realización de evaluaciones en los procesos de oposición y selección del profesorado de Educación Física en las etapas de Primaria y Secundaria. Esto puede ser especialmente relevante en unos momentos en que el Ministerio de Educación está planteando cambiar radicalmente los procesos de selección del profesorado de las etapas obligatorias, estableciendo el denominado en algunos foros como: "MIR educativo" (López-Rupérez, 2015; Marina, Pellicer y Manso, 2015).

No quisiéramos terminar sin señalar algunas limitaciones que presenta nuestra propuesta. Algunas preguntas de la escala pudieran no estar adecuadamente enunciadas, pues la interpretación que han mostrado los estudiantes y egresados no se ajusta a lo esperado. Concretamente, la pregunta Conocer y promover las diferentes manifestaciones motrices que forman parte de tu cultura tradicional, que el análisis factorial asignaba al F2, cabría esperar que, por su contenido, se asignara al factor F3. Cabe señalar, además, la necesidad de aumentar la fiabilidad de las subescalas correspondientes a los factores F2, F3 y F4, pues, pese a estar dentro de los valores admisible psicométricos, están por debajo de los obtenidos por la prueba en su conjunto o en la subescala del factor F1. Las líneas a seguir para solventar este problema será el añadir competencias que complementen estos tres factores.

Por último, señalamos algunas de las líneas de trabajo futuras entre las que cabe señalar la solución de las limitaciones señaladas en el párrafo anterior. Asimismo, toda vez que se dispone de un instrumento robusto, se abre un interesante línea de investigación en la comparación de la percepción de competencias de los recién ingresados y de los recién egresados.

\section{REFERENCIAS BIBLIOGRÁFICAS}

Akaike, H. (1987). Factor analysis and AIC. Psychometrika. 52, 317-332. https://doi.org/10.1007/BF02294359

ANECA. (2005a). Libro Blanco. Título de Grado en Ciencias de la Actividad Física y el Deporte. Madrid: Agencia nacional de Evaluación de la Calidad y la Acreditación.

ANECA. (2005b). Libro Blanco. Título de Grado en Magisterio. Madrid: Agencia nacional de Evaluación de la Calidad y la Acreditación.

Aparicio, J. L. y Fraile, A. (2015). La evaluación de competencias interpersonales en la formación del profesorado de Educación Física a través de un programa de expresión corporal. International Journal for 21st Century Education (IJ21CE), 2(2), 21-34

Baena, A. y Granero, A. (2012). Competencias profesionales en Educación Física y necesidades educativas. Espiral. Cuadernos del Profesorado, 5(10), 105-109. https://doi.org/10.25115/ecp.v5i10.945 
Baena A., Granero A., y Martínez, M. (2015). Validación española de la Escala de Evaluación de la Competencia Docente en Educación Física de secundaria. Cuadernos de psicología del deporte, 15(3), 113-122. https://doi.org/10.4321/S1578-84232015000300011

Batista, J. M. y Coenders, G. (2000). Modelos de Ecuaciones Estructurales. Madrid: La Muralla.

Bentler, P. M. (1990). Comparative fit indices in structural models. Psychological Bulletin, 107, 238-246. https://doi.org/10.1037/0033-2909.107.2.238

Bentler, P. M. y Bonnet, D. C. (1980). Significance Tests and Goodness of Fit in the Analysis of Covariance Structures, Psychological Bulletin, 88 (3), 588606. https://doi.org/10.1037/0033-2909.88.3.588

Boned, C. J., Rodríguez-Romo, G., Mayorga, J. I. y Merino, A. (2006). Competencias profesionales del Licenciado en Ciencias de la Actividad Física y del Deporte, Motricidad, 15, 1-6.

Browne, M. W., y Cudeck, R. (1993). Alternative ways of assessing model fit. In: K. A. Bollen \& J. S. Long (Eds.), Testing structural equation models (pp. 136-162). Beverly Hills, CA: Sage.

Carretero-Dios, H. y Pérez, C. (2005). Normas para el desarrollo y revisión de estudios instrumentales. International Journal of Clinical and Health Psychology, 5 (3), 521-551.

Castejón-Oliva, F. J.; Santos-Pastor, M. L. y Palacios-Picos, A. (2015) Cuestionario sobre metodología y evaluación en formación inicial en educación física. Revista Internacional de Medicina y Ciencias de la Actividad Física y el Deporte, 15 (58), 245-267. https://doi.org/10.15366/rimcafd2015.58.004

Catano, V. M. y Harvey, S. (2011). Student perception of teaching effectiveness: development and validation of the Evaluation of Teaching Competencies Scale (ETCS). Assessment \& Evaluation in Higher Education, 36 (6), 701 717. https://doi.org/10.1080/02602938.2010.484879

Corominas, E. (2001). Competencias genéricas en la formación universitaria. Revista de Educación, 325, 299-321.

Corominas, E., Tesauro, M., Capell, D., Teisidó, J., Pèlach, J., y Cortada, R. (2006). Percepciones del profesorado ante la incorporación de las competencias genéricas en la formación universitaria. Revista de Educación, 341, 301-336.

Delors, J. (1996). La educación encierra un tesoro: informe a la UNESCO dela Comisión Internacional sobre la Educación para el Siglo XXI. Madrid: Santillana.

Díaz del Cueto, M. (2009). Percepción de competencia del profesorado de Educación Física e inclusión. Revista Internacional de Medicina y Ciencias de la Actividad Física y el Deporte, 9 (35) pp. 322-348 http://cdeporte.rediris.es/revista/revista35/artpercepcion152.htm

Fraile, A. (2004). Un cambio democrático en las aulas universitarias: una experiencia en la formación del profesorado de Educación Física. Contextos educativos (6-7), 213-234. https://doi.org/10.18172/con.537

Fraile, A. y Aparicio, J. L. (2015a). Las competencias interpersonales que deben estar presentes en la formación inicial del profesorado de educación física. Acción, 11(21), 25-30. 
Fraile, A. y Aparicio, J. L. (2015b). Expresión corporal y el desarrollo de competencias transversales en la formación del profesorado de educación física. Tándem, 47, 1-8.

Gallardo, M. A. (2006). Evaluación de las competencias profesionales para la inserción laboral de los maestros de Educación Física. Revista Electrónica de Investigación Psicoeducativa, 9(3), 469-492.

Gallego-Ortega, J. L. y Rodríguez-Fuentes, A. (2019) Percepciones del profesorado sobre competencias comunicativas de futuros maestros de educación. Revista Internacional de Medicina y Ciencias de la Actividad Física y el Deporte, 19 (75), en prensa.

Garmendia, C. (2009). De la construcción del Espacio Europeo de Educación Superior, «Bolonia» y otros demonios. La Cuestión Universitaria, 5, 4-9. https://doi.org/10.3989/arbor.2009.extran1202

Hernández, J. L. (2007). El nuevo modelo de maestro. Competencias de la Mención de Educación Física. En P. Palou, F.J. Ponseti, P. A Borrás, y J. Vidal (Coord). Educación Física en el Siglo XXI. Nuevas perspectivas Nuevos retos (163-183). Palma de Mallorca: Universitat de les Illes Balears.

Le Boterf, G. (2000). Ingeniería de las competencias. Barcelona: Gestión.

Lleixá, T; Robert, M. y Batalla, A. (2010). Evaluación de competencias en la formación del profesorado de Educación Física: El caso del Blaagaard Seminarium de Copenhague. Fuentes, 8, 116-124.

Lleixá, T; Torralba, M. A y Abrhao, S. R. (2010). Evaluación de competencias en Educación Física: Investigación-acción para el diseño de procedimientos de evaluación en la Etapa Primaria. Movimento, 6(4), 33-51.

López-Rupérez, F. (2015). MIR educativo y profesión docente. Un enfoque integrado. Revista Española de Pedagogía, 261 (73-2), 283-299.

Marchesi, A. (2005). Sobre el bienestar de los docentes. Madrid: Alianza Editorial.

Marina, J. A; Pellicer, C y Manso, J, (2015). Libro Blanco de la profesión docente y su entorno escolar. Madrid: MEC. Recuperado de https://dialnet.unirioja.es/servlet/libro?codigo=581104\&orden=1\&info=op en link libro

Martín, E. y Moreno, A. (2007). Competencia para aprender a aprender. Madrid: Alianza Editorial.

Martínez-Mínguez, M. L. (2016). Proyectos de aprendizaje tutorados y autoevaluación de competencias profesionales en la formación inicial del profesorado. Retos: nuevas tendencias en educación física, deporte y recreación, 29, 242-250.

MECD (2003). La integración del sistema universitario en el Espacio Europeo de Educación Superior. Documento-Marco. Madrid: Ministerio de Educación, Cultura y Deporte.

Moreno-Murcia, J. A.; Silveira, Y. y Belando, N. (2015). Questionnaire evaluating teaching competencies in the university environment. Evaluation of teaching competencies in the university. New Approaches In Educational Research, 4-1, 54-61. https://doi.org/0.7821/naer.2015.1.106

Navio, A. (2005). Propuestas conceptuales en torno a la competencia profesional. Revista de Educación, 337, 213-234. 
Olsson, V. (1979). Maximum Likehood Estimation of the Polichoric Correlation Coefficient. Psychometrika, 44, 443-460. https://doi.org/10.1007/BF02296207

OXFAM NOVID (2011). Educadores de calidad: Estudio internacional sobre las competencias y estándares para docentes. Bruselas: Internacional de la Educación.

Palmer, A; Montaño, J. J. y Palou, M. (2009). Las competencias genéricas en la educación superior. Estudio comparativo entre la opinión de empleadores y académicos. Psicothema, 21, 3, 433-438.

Pereda, S. y Berrocal, F. (1999). Gestión de recursos humanos por competencias. Madrid. Centro de Estudios Ramón Areces.

Perrenoud, P. (2004). Diez nuevas competencias para enseñar. Barcelona: Graó.

Romero, C. (2009). Definición de módulos y competencias del maestro con mención en Educación Física. Revista Internacional en Medicina de Ciencias de la Actividad Física y el Deporte, 9 (34), 179-200.

Rué, J. (2007). Enseñar en la universidad: el EEES como reto para la educación superior. Narcea, Madrid.

Salcines-Talledo, I., González-Fernández, N., Ramírez-García, A. y MartínezMínguez, L. (2018). Validación de la Escala de Autopercepción de Competencias Transversales y Profesionales de Estudiantes de Educación Superior. Profesorado. Revista de Curriculum y formación del Profesorado, 22(3), https://doi.org/10.30827/profesorado.v22i3.7989

Solanes, A., Núñez, R. y Rodríguez, J. (2008). Elaboración de un cuestionario para la valoración de competencias genéricas en estudiantes universitarios. Apuntes de Psicología, 26 (1), 35-49.

Tejada, J. (2005). El trabajo por competencias en el prácticum: cómo organizarlo y cómo evaluarlo. Revista Electrónica de Investigación Educativa, 7(2), 131 Recuperado de http://redie.uabc.mx/vol7no2/contents-tejada.html

Zabalza, M. A. (2003). Competencias docentes del profesorado universitario. Calidad y desarrollo profesional. Madrid: Narcea.

Número de citas totales / Total references: 47 (100\%)

Número de citas propias de la revista / Journal's own references: $4(8 \%)$

Rev.int.med.cienc.act.fís.deporte - vol. 19 - número 75 - ISSN: 1577-0354 